\title{
Knowledge, attitudes and practice of preoperative fasting guidelines in the National Hospital of Sri Lanka
}

\author{
Gunawardhana S. A. A. I \\ Senior Registrar in Anaesthesiology, National Hospital of Sri Lanka
}

\begin{abstract}
Background
Recent guidelines on preoperative fasting recommend 2 hours for clear fluids and 6 hours for solids in most elective surgical patients ${ }^{1,2}$. Despite this contemporary practice varies widely across the country. This study evaluates the knowledge, current practices and compliance with guidelines.
\end{abstract}

\section{Method}

This was a cross-sectional study. Data was obtained from patients attending for elective surgeries in NHSL during July 2010 and from junior anaesthetists, intern medical officers (IMO) and nurses of surgical wards. ASA practice guideline was the standard of assessment.

\section{Results}

235 patients and 118 healthcare workers were included. The mean duration of fasting was 13.86 hours (range $8-18$ ) for solids and 12.68 hours for liquids $(4-18)$. All junior anaesthetists and $64.3 \%$ of IMOs had above satisfactory level of knowledge. Nurses had below satisfactory knowledge. Only $58.3 \%$ of anaesthetists and none of the nurses or IMOs have adhered to guidelines. $81 \%$ patients stated that they would refuse a meal at 2 am but $66 \%$ would have appreciated a cup of tea 2 hours before surgery.

\section{Conclusion}

Patients undergoing routine surgery fast for unnecessarily long periods. Failure of implementation of guidelines is mainly due to inadequate knowledge and poor attitude among ward staff. More pragmatic approach is needed to assure that fasting instructions are consistent with the ASA guidelines and that patients understand and comply with these directives.

Key words: preoperative fasting, knowledge, practice

\section{Introduction}

Prolonged period of preoperative fasting has been the accepted practice until recently and "nil per oral" after midnight of the day before surgery was recommended in the late 1940's when a high incidence of pulmonary aspiration of gastric contents was discovered among obstetric patients undergoing general anaesthesia ${ }^{3}$. Recent research show that pulmonary aspiration occurs only rarely as a complication of modern general anaesthesia and in fact prolonged fasting causes several adverse effects such as increased patient anxiety, discomfort and irritability, dehydration, hypovolaemia and hypoglycemia ${ }^{1}$. The benefits of reduced preoperative fasting, increased patient comfort and hydration, coupled with an unchanged risk of adverse events has led several of the leading societies, i.e. American Society of Anesthesiologists' (ASA), Association of Anaesthetists of Great Britain and Ireland (AAGBI) to develop new guidelines supporting more liberal preoperative fasting times guidelines.

The ASA practice guidelines for preoperative fasting in healthy patients undergoing elective procedures, revised in 1999, recommend a fasting period of 2 hours for clear fluids, 6 hours for solids allowing a light meal 6 hours before and a heavier meal 8 hours before the procedure $^{1}$. Based on this the Ministry of Health of Sri Lanka in collaboration with the College of Anaesthesiologists of Sri Lanka developed a national guideline regarding preoperative preparation including preoperative fasting under the World Bank project. Despite evidence that shortened preoperative fasts does not increase 
the risk of a harmful event for the patient, contemporary practice still has wide variations across the country.

Audits carried out in the west have discovered that implementation of these new guidelines tends to lag and that unnecessarily prolonged preoperative fasting is still the norm rather than the exception in most centers. It has been observed that this remains an issue here as well.

\section{Methodology}

The study is a cross-sectional survey carried out in the National Hospital of Sri Lanka, a tertiary care unit with approximately 3000 beds, during the months of June and July in 2010.

The Director of the institution and the consultants of the relevant surgical wards were informed and their express consent for conducting the audit was obtained. Ethical approval for conducting the audit was obtained from the ethics committee of the National Hospital of Sri Lanka.

Study participants

- Junior anaesthetists (registrars and medical officers of anaesthesiology) assessing the patients preoperatively, intern medical officers and nurses of the relevant surgical wards

- Non obstetric 'healthy' patients (ASA I-II without gastrointestinal disease or disorders) who are aged eighteen years or more, presenting for elective surgeries

235 patients and 118 healthcare workers were included in the study.

Patients who require specific bowel preparation for surgical reasons (e.g. - gastrointestinal surgery) and patients who are expected to be at increased risk of regurgitation and aspiration, such as those who have gastro-oesophageal reflux, gastric outlet/ intestinal obstruction, obesity and diabetes were excluded.

Patients and health care workers who were not willing to participate were not included in the study.

Data was collected from the bed head tickets and directly from the patients by an interviewer administered questionnaire. A separate self administered questionnaire was given to the health care workers. Both questionnaires were pretested and expert validated.

National guidelines and ASA practice guidelines for preoperative fasting and the use of pharmacologic agents to reduce the risk of pulmonary aspiration: application to healthy patients undergoing elective procedures published in 1999 was the standard of assessment.

Data analysis was done using the computer software SPSS version 14(SPSS Inc. Chicago, IL, USA).

\section{Results}

Two hundred and thirty five patients presenting for elective surgery [male: female $=108(46.8 \%)$ : $127(53.2 \%)$, mean age $=45.17$ years] were recruited. Most patients were educated only up to $\mathrm{GCE} \mathrm{O/L}$ with only $2.1 \%$ of the population having undergone higher education. Majority $(74.5 \%, \mathrm{n}=175)$ of patients did not have any significant co- morbidities (ASA group 1). Most patients $(36.2 \%, \mathrm{n}=87)$ had undergone grade 2(intermediate) surgical procedures. $63.8 \%(n=$ 149) of the surgeries were done under general anaesthesia.

One hundred and eighteen health care workers (anaesthetists-50, intern medical officers-32, nurses-36) were also included in the study.

Table 1:Characteristics of the patient population

\begin{tabular}{|l|l|l|}
\hline Characteristic & $\begin{array}{l}\text { Number of } \\
\text { patients }\end{array}$ & Percentage \\
\hline Gender & \multicolumn{3}{|c|}{} \\
Male & 108 & $46.8 \%$ \\
female & 127 & $53.2 \%$ \\
Education level & & \\
Up to grade 8 & 74 & $31.9 \%$ \\
Up to GCE O/L & 83 & $34.0 \%$ \\
Up to GCE A/L & 74 & $31.9 \%$ \\
Higher education & 4 & $2.1 \%$ \\
ASA group & & \\
Grade I & 175 & $74.5 \%$ \\
Grade II & 60 & $25.5 \%$ \\
\hline Surgical grading & & \\
Grade 1 & 74 & $31.9 \%$ \\
Grade 2 & 87 & $36.2 \%$ \\
Grade 3 & 74 & $31.9 \%$ \\
Grade 4 & 0 & $0 \%$ \\
\hline
\end{tabular}




\begin{tabular}{|lll|}
\hline Type of anaesthesia & & \\
General anaesthesia & 149 & $63.8 \%$ \\
Spinal anaesthesia & 70 & $29.8 \%$ \\
Epidural anaesthesia & 0 & $0 \%$ \\
Regional nerve blocks and & 16 & $6.4 \%$ \\
local anaesthesia & & \\
\hline
\end{tabular}

On $72.3 \%(n=170)$ of the bed head tickets (BHT) fasting instructions were recorded by the intern medical officers, and on $78.7 \%(n=185)$ and $95.7 \%(n=225)$ of BHTs, by anaesthetists and nurses respectively. The recorded instructions are shown in Table 2.

On $95.6 \%$ patients, nurses advocated fasting from $10 \mathrm{pm}$ and they gave the last instruction regarding preoperative fasting to all patients prior to start of list.

Table 2: Instructions recorded on BHT by health care workers

\begin{tabular}{|l|l|l|l|}
\hline Instruction & $\begin{array}{l}\text { Intern medical } \\
\text { officers (IMO) }\end{array}$ & Anaesthetists & Nurses \\
\hline $\begin{array}{l}\text { Fasting from } \\
\text { 10pm }\end{array}$ & $91.2 \%$ & $16.7 \%$ & $95.6 \%$ \\
$\begin{array}{l}\text { Fasting from } \\
\text { midnight }\end{array}$ & $5.9 \%$ & $11.1 \%$ & $2.2 \%$ \\
$\begin{array}{l}\text { Fasting for 6 } \\
\text { hours }\end{array}$ & $2.9 \%$ & $8.3 \%$ & $2.2 \%$ \\
$\begin{array}{l}\text { Fasting 6 hours } \\
\text { for solid/2 hours } \\
\text { for clear fluid } \\
\text { fasting 6 hours } \\
\text { for solids and } \\
\text { plain tea at 6 }\end{array}$ & $0 \%$ & $58.3 \%$ & $0 \%$ \\
a.m. & & $5.6 \%$ & $0 \%$ \\
\hline
\end{tabular}

$63.8 \%$ of patients had been verbally instructed by anaesthetists regarding preoperative fasting and $53.2 \%$ and $97.9 \%$ by IMOs and nurses respectively.

The actual mean duration of preoperative fasting the patients had adhered to until the start of the list at 8 am for water/ clear fluids was 10.37 hours (range 3-14 hrs) and for light or heavy meals was $11.6 \mathrm{hrs}$ (range $8-14 \mathrm{hrs}$ ). The duration of fasting until the start of the surgery is shown below.
Table 3: Duration of fasting until start of the surgery

\begin{tabular}{|l|l|l|}
\hline & Mean & Range \\
\hline Water/ clear fluids & $12.86 \mathrm{hrs}$ & $4-18 \mathrm{hrs}$ \\
Solids (light/ heavy meal) & $13.86 \mathrm{hrs}$ & $8-18 \mathrm{hrs}$ \\
\hline
\end{tabular}

$70.2 \%$ of patients stated that they felt discomfort during the preoperative period and factors contributing to discomfort were thirst (57.6\%), hunger $(48.5 \%)$, anxiety (63.6\%), lengthy wait prior to surgery $(15.2 \%)$, and nausea/vomiting $(12.1 \%)$. The relative severity of hunger and thirst felt by patients was subjectively assessed.

Table 4: Severity of hunger/thirst felt by patients

\begin{tabular}{|l|l|l|l|l|}
\hline & $\begin{array}{l}\text { No } \\
\text { hunger/thirst }\end{array}$ & mild & moderate & severe \\
\hline Hunger & $46.8 \%$ & $23.4 \%$ & $25.5 \%$ & $4.3 \%$ \\
Thirst & $34 \%$ & $31.9 \%$ & $27.7 \%$ & $6.4 \%$ \\
\hline
\end{tabular}

$66 \%$ patients said that they would have liked a cup of plain tea at 6 am on the day of surgery but only $19.1 \%$ would have taken a light meal at 2 am (6 hrs prior to the start of the list). Reluctance to wake up at 2 am $(71.1 \%)$, fear of vomiting $(39.5 \%)$, fear of wanting to pass urine $(15.8 \%)$ and fear of surgery being postponed $(36 \%)$ were the reasons given for this.

$70 \%$ of health care workers were aware of at least one of the recommended guidelines (ASA, AAGBI, RCN, National guidelines) but only $37.5 \%$ were aware of the national guidelines.

The knowledge of health care workers regarding the preoperative fasting according to guidelines was assessed using a pre validated knowledge questionnaire and scoring system. Each response was allocated one mark giving a total of sixteen and knowledge was graded as poor (0-4), unsatisfactory (5-8), satisfactory (9-12) or good (13-16) according to the marks obtained out of sixteen. All junior anaesthetists and $64.3 \%$ of IMOs had above satisfactory level of knowledge. The knowledge of $66.6 \%$ of nurses was either poor or unsatisfactory.

Majority $(n=77,65 \%)$ of health care workers who had satisfactory or good knowledge of fasting guidelines accepted that they did not 
implement them in practice and the reasons given are shown in table 4. Only $48.6 \%$ encouraged patients to take clear fluids up to 2 hours prior to the start of the list.

Table 5: Reasons given by health care workers for not implementing guide lines

\begin{tabular}{|l|l|l|}
\hline & $\mathrm{n}$ & $\%$ \\
\hline $\begin{array}{l}\text { Feel that patients will not understand } \\
\text { instructions properly }\end{array}$ & 24 & $45.9 \%$ \\
Unit policy differs from guidelines & 17 & $21.6 \%$ \\
$\begin{array}{l}\text { Senior advice differs from guidelines } \\
\text { Feel that fasting time according to }\end{array}$ & 19 & $24.3 \%$ \\
guidelines inadequate & 0 & $0 \%$ \\
Inadequate time due to high work load & 17 & $21.6 \%$ \\
& & \\
\hline
\end{tabular}

\section{Discussion}

The aim of this study was to assess the practice of preoperative fasting guidelines in a Sri Lankan setting. It is evident from the study that almost all patients undergoing elective surgery in NHSL are subjected to unnecessarily prolonged preoperative fast causing distress and discomfort to them. Although the doctor's (anaesthetists and IMOs) knowledge of current guidelines is satisfactory the knowledge of nurses who provide verbal instructions last is poor. Furthermore fasting instructions are given by more than one healthcare worker to a patient and in most instances the medical advice was overridden by nurses instructions. This contributes significantly to failure of implementation of guidelines. Even those health care workers with satisfactory knowledge and awareness of guidelines tend not to practice them. Although most patients felt mild to moderate hunger and thirst, they were not enthusiastic about taking a meal six hours prior to start of the morning list(at 2.00am). However most patients stated that they would have been more comfortable, had they been allowed clear fluids in the morning two hours prior to the start of the list.

\section{Recommendations}

More pragmatic approach is needed to assure that fasting instructions are consistent with the ASA/national guidelines and that patients understand and comply with these directives.
Health care workers should be educated regarding the guidelines and the importance of adherence to them. Greater emphasis should be on educating the nurses on the guidelines and on not over riding the instructions of medical staff.

Doctors should verbally instruct the patients as well as the nurses regarding the correct fasting regime. Patients should be actively encouraged to take clear fluids up to two hours prior to start of the list.

\section{References}

1. Practice guidelines for preoperative fasting and the use of pharmacologic agents to reduce the risk of pulmonary aspiration: Application to Healthy Patients Undergoing elective Procedures Anesthesiology.1999;90:896-905

2. Pre-operative assessment, the role of the anaesthetist. Association of Anaesthetists of Great Britain and Ireland November 2001. 3. Preoperative fasting for adults to prevent perioperative complications. The Cochrane Database of Systematic Reviews 2003, Issue 4. 4. Perioperative fasting in adults and children - an $\mathrm{RCN}$ guideline for the multidisciplinary team. RCN publications November 2005. 5. A national survey on preoperative fasting policies and practices in Jamaican hospitals. McGawCD, Ehikhametalor E, Nelson M, Soogrim D. West Indian MedJ.2004Sep;53(4):227-33.

PMid:15622675 